\title{
Rectal Carcinoma, Recent Staging Strategy by MRI Using Diffusion Weighted Sequence
}

\author{
Waleed Abdelfattah Mousa, Tarek Fawzy Abdella \\ Department of Diagnostic Radiology, Menoufia University, Menoufia, Egypt \\ Email address: \\ waleedmousa75@gmail.com (W. A. Mousa),tarekvip100@yahoo.com (T. F. Abdella) \\ To cite this article: \\ Waleed Abdelfattah Mousa, Tarek Fawzy Abdella. Rectal Carcinoma, Recent Staging Strategy by MRI Using Diffusion Weighted Sequence. \\ International Journal of Medical Imaging. Vol. 5, No. 2, 2017, pp. 19-25. doi: 10.11648/j.ijmi.20170502.13
}

Received: December 19, 2016; Accepted: January 3, 2017; Published: March 25, 2017

\begin{abstract}
Objective: MRI is currently the imaging modality of choice for the detection, characterization, and staging of rectal cancer. A variety of examinations have been used for preoperative staging of rectal cancer, including digital rectal examination, endorectal (endoscopic) ultrasound, CT, and MRI. Endoscopic ultrasound is the imaging modality of choice for small and small superficial tumors. MRI is superior to CT for assessing invasion to adjacent organs and structures, especially low tumors that carry a high risk of recurrence. Background: Colorectal cancer (CRC) is the third most common cancer in both sexes combined worldwide, after prostate and breast cancer with an incidence of 40 in 100,000. Rectal carcinoma accounts for more than one-third of colorectal tumors and is associated with significant morbidity and mortality. Currently, MRI using diffusion weighted sequence is the most sensitive and specific modality in staging rectal cancer as it is able to depict the mesorectal fascia and its relation to the tumor margins precisely. Methods: This study included 50 patients, (32 male and 18 female) previously diagnosed as cancer rectum based on proctoscope and histopathological biopsy. The age range of the patients was from 18 years to 78 years. All patients will be subjected to the following: detailed history, clinical examination, proctoscopic assessment and histopathological data. All patients underwent MRI of the pelvis specifically cancer rectum protocol. Results: The most frequently encountered clinical presentation among cancer rectum patients was bleeding per rectum (14), constipation (26), incidentally discovered liver metastasis (6) and intestinal obstruction (4). MRI evaluation of $T$ staging in correlation to the histopathological examination showed sensitivity (80\%), specificity (93\%), accuracy (94\%). Lymph node (LN) metastatic spread was evaluated by MRI and showed sensitivity (87\%), specificity (92\%), accuracy (92.6\%), PPV (84.8\%) and NPV (96.7\%). Regarding circumferential resection margin (CRM) MRI assessment revealed; sensitivity (96\%), specificity (94\%), accuracy (96\%), PPV (95\%) and NPV (94.3\%). Conclusions: MRI is an accurate and sensitive imaging method delineating tumoral margins, mesorectal fascia involvement, lymph nodes, and distant metastasis. MRI can accurately delineate the mesorectal fascia involvement, which is one of the main decision points in planning treatment.
\end{abstract}

Keywords: Magnetic Resonance Imaging, Diffusion, Cancer Rectum, Tumor Staging

\section{Introduction}

Colorectal carcinoma is the third most common cancer among both sexes combined worldwide, after prostate and breast cancer with an incidence of 40 per 100,000 [1]. Cancer rectum accounts for more than one third of colorectal tumors and is associated with significant morbidity and mortality [2].

Rectal tumor staging is commonly performed using various modalities such as computed tomography (CT) scan, endoscopic ultrasound (EUS) and MRI [3].

Currently, MRI is the most sensitive and specific modality in staging rectal cancer as it is able to precisely depict the mesorectal fascia relation to the tumor margins due to its superior spatial and tissue contrast resolution [4].

To identify and stage rectal cancers with MRI, high resolution T2-weighted image with thin-sections is the primary approach used to differentiate between the tumor, mucosal, submucosal layers, muscular layer, perirectal fat and mesorectal fascia (MRF) [5,6]. MRI also improves the assessment of nodal morphology [7].

Rectal cancer staging is based on two principles. The first is defining the pertinent anatomy allowing for surgical planning. The second is to allow prognostic stage grouping. The aim of preoperative staging is to accurately and 
reproducibly differentiate between good and poor prognosis tumors. Prognostic stratification includes assessment of depth of spread, nodal status, extramural vascular invasion (EMVi), CRM and peritoneal invasion [8].

The aim of this work is to show the current staging strategy of rectal carcinoma by MRI.

\section{Methods}

Fifty patients with primary rectal cancer were studied (32

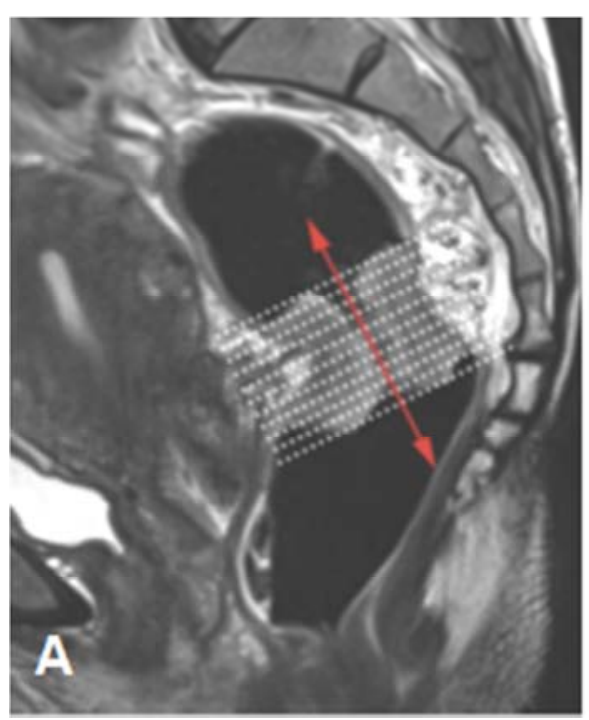

males and 18 females). The age range was 18 to 78 years with mean age 48 years.

The diagnosis of rectal carcinoma in these patients was established based on their proctoscopy, biopsy and histopathology. The lesions were located $4-16 \mathrm{~cm}$ from the anal verge.

Pelvic MRI examination was performed to all patients using 1.5 T magnet (Philips-Achieva) with pelvic phased array coil.

MRI Protocol

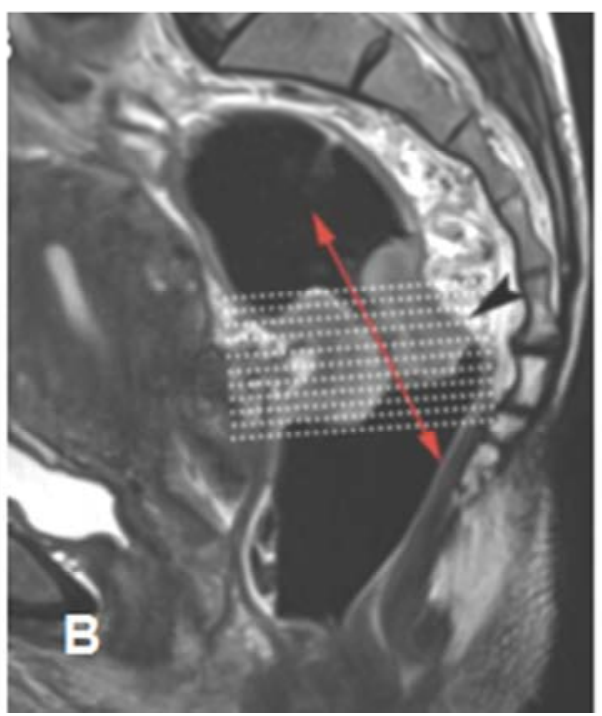

Figure 1. Sagittal T2-weighted image used for planning high-resolution T2 sequence. (A) Plane of planned images (white dotted lines) is perpendicular to the axis of rectum/tumor (red arrow [A, B]). Incorrect selection of imaging plane (B) results in poor delineation of muscular layer due to averaging through a plane oblique to the tumor and rectum (arrowhead [B]).

Our MRI protocol consists of sagittal T2-weighted singleshot images and T2-weighted turbo spin-echo (TSE) images in the axial and coronal planes. Unenhanced and contrastenhanced axial and coronal T1-weighted fat-saturated images of the rectum are also obtained. We start by sagittal T2weighted TSE sequence to locate the tumor. On the basis of the sagittal sequence, axial and coronal T2-weighted images, TSE sequences are planned, and they are angled to the plane exactly perpendicular and parallel to the tumor axis (figure 1). Axial images of the tumor are important because they reduce the overestimation of the tumor depth of invasion noted on oblique imaging [9]. Coronal images help in identifying the relationship of low rectal tumors to the internal and external sphincter [10]. T2-weighted sagittal images help assess the relationship of the tumor to the peritoneal reflection (figure 2) .

The addition of diffusion weighted images (DWIs) to T2weighted images improves accuracy for rectal cancer detection. Images are acquired in the axial plane with the patient in free breathing from the level of the aortic bifurcation to the upper thigh in order to include inferior mesenteric lymph nodes and groin nodes. Diffusion gradients at 4 time points (or b values) are obtained (b values 0,50 , 400, and 800). An apparent diffusion coefficient (ADC) map is subsequently generated using all $b$ values. Low $b$-value images (0 and 50) provide maximal lesion detection particularly for the presence of lymph nodes and bone metastases, whereas high b-value images (b value 800 ) provide signal suppression of highly cellular structures such as gastrointestinal and urogenital lining to maximize conspicuity of tumor [12] (figure 3).

The high signal intensity focus depicting a tissue with restricted diffusion is readily apparent against a low signal intensity background of bowel wall and feces on high b-value images. Hence, the sequence generally aids in detection of small tumors not seen on T2-weighted images. DWI has several limitations. Benign tumors may show restricted diffusion. Hyperintensity due to collapsed bowel wall may also mimic disease \& also, the lower spatial resolution of DWI sequences [11].

Operative data and postoperative pathological staging were compared to the MRI staging.

Results of MRI and histopathology were collected and compared in a single chart and statistical analysis was done manually.

\section{Results}

The fifty patients included in this study showed different pathological types of cancer rectum, 23 patients were diagnosed as adenocarcinoma, 22 as mucinous carcinoma while the tumor was undifferentiated in 5 patients. 
Table 1. Number and percentage of different pathological species.

\begin{tabular}{lll}
\hline Pathological type & Number & Percent \\
\hline Well differentiated adenocarcinoma & 23 & $46 \%$ \\
Mucinous carcinoma & 22 & $44 \%$ \\
Poorly differentiated /Undifferentiated type & 5 & $10 \%$ \\
Total & 50 & $100 \%$ \\
\hline
\end{tabular}

The location of the tumor in the rectum and its distance from the anal verge are represented in table (2):

Table 2. Location of the rectal lesions and the number of patients at each site.

\begin{tabular}{ll}
\hline Tumor location & No. of cases \\
\hline Upper third of the rectum "12-16cm from the anal verge" & 15 \\
Middle $1 / 3$ of the rectum "8-11cm from the anal verge" & 14 \\
lower $1 / 3$ of the rectum "4-7cm from the anal verge" & 17 \\
Whole rectal length or extended to more than one third & 4 \\
Total & 50 \\
\hline
\end{tabular}

$\mathrm{T}$ staging of rectal cancer by MRI using the four layer anatomic model revealed 4 patients in stage T1, 3 of them were confirmed pathologically and one proved to be in stage T2. Six were diagnosed as stage T2 cancer rectum as there was invasion of the rectal layer up to the muscularis propria with no penetration of the muscularis propria or perirectal fat. Histopathological examination confirmed the MR finding in 4 patients, but diagnosed one as stage T3 and another one as stage T3. In 27 patients there was invasion of all rectal layers as will as perirectal fat infiltration yet without extension to the pelvic organs thus the MRI diagnosis was stage T3. This was confirmed by histopathological examination in 23 patients, but 2 were in stage T4A, one in stage $\mathrm{T} 2$ and the last in stage $\mathrm{T} 1$. Thirteen patients showed pelvic organs and/or peritoneal fold invasion thus were considered as stage T4. This was confirmed by histopathological examination in 12 patients, but one patient was in stage T3. MRI shows $80 \%$ sensitivity, $93 \%$ specificity and $94 \%$ accuracy in evaluation of $\mathrm{T}$ staging in comparison to the histopathological examination.

Table 3. Comparison of staging by MRI and histopathological results.

\begin{tabular}{llllll}
\hline MR & \multicolumn{2}{l}{ Histopathology } & & & \\
\hline & T0 & T1 & T2 & T3 & T4 \\
\hline T0 & - & - & - & - & - \\
T1 & - & 3 & 1 & - & - \\
T2 & - & 1 & 4 & 1 & - \\
T3 & - & 1 & 1 & 23 & 2 \\
T4 & - & - & - & 1 & 12 \\
\hline
\end{tabular}

In 27 patients MRI showed no mesorectal LN involvement, and this was confirmed pathologically in 25 patients, while two patients had positive metastatic regional perirectal lymph nodes. MRI showed regional lymph node involvement in 23 patients; twenty in stage N1 and three in stage N2. This was confirmed histopathologically in 22 patients, while one patient was found to be in stage N1 instead of N2. The total number of recorded malignant lymph nodes was 54 divided between 42 mesorectal, 6 internal iliac and 4 common iliac nodes.

Lymph node metastatic spread evaluated by MRI showed
$87 \%$ sensitivity, $92 \%$ specificity and $92.6 \%$ accuracy.

The CRM was involved by MRI in thirty one patients, 30 of them were confirmed pathologically. 19 patient MRI had free CRM, one of them was proved pathologically to have involved CRM. Regarding CRM assessment, the study revealed 96\% sensitivity, 94\% specificity, 94\% accuracy, 95\% PPV and 94.3\% NPV measures 94\%, 96\%, 95\% and $94.3 \%$ respectively.

\section{Discussion}

Three main modalities are used in preoperative staging of rectal cancer: CT, EUS and MRI. CT was the first modality developed and proved useful in demonstrating advanced disease and distant metastases but less useful in determining the degree of tumor extension through the bowel wall and local lymph node metastases. With the development of EUS the extent of tumor invasion and perirectal lymph node metastases can be evaluated, although distant nodal involvement can't be assessed. MRI nowadays can be used to assess wall penetration, perirectal nodal involvement and distant metastases [9].

In this study, MRI T-staging matched with histological Tstaging in 43 out of 50 patients ( $86 \%$ accuracy), and mismatched in seven due to the difficulty in differentiation between stages T2 \& T3. these results showed $88.8 \%$ sensitivity, $96.5 \%$ specificity, and $92.1 \%$ accuracy. The results of Algebally et al. [14] showed that MRI T-staging matched with histological T-staging in 48 out of 56 patients (85.7\% accuracy), and mismatched in eight patients.

Elizabeth Furey et al. [12] reported that with using high resolution MRI, they achieved accuracy more than $85 \%$ in $\mathrm{T}$ staging.

In a prospective study done by Rao S-X, et al. [13] including 67 patients with primary rectal cancer, the overall magnetic resonance accuracy for T staging was $85.1 \%$.

Matsuoka et al. [15] reported different results that do not match with ours; $100 \%$ accuracy, sensitivity and specificity of MRI in T staging. This was probably due to small sample size of their study, only 21 patients.

The assessment of nodal involvement remains a confounding factor. Cross-sectional imaging relies on size as a criterion for nodal involvement, which has significant limitations. High resolution MR imaging allows the assessment of nodal morphology, which significantly improves specificity in the assessment of nodal involvement. DWI is a useful adjunct to conventional T2 imaging, increasing the sensitivity of small lymph nodes. [18]

In our study the specificity, sensitivity and accuracy of MRI in detection of lymph node involvement was $87 \%, 92 \%$, $92.6 \%$ respectively.

Zhang et al. [18] reported lower values (79\% specificity, $64.7 \%$ sensitivity and $90.5 \%$ accuracy). This might be because in our study we relied upon morphological criteria of the lymph nodes as well as the diffusion pattern and its ADC value, and those additional criteria improve results of the nodal assessment. 
Wong et al. [16] studied 50 patients and reported $80 \%$ sensitivity and $86 \%$ accuracy in diagnosis of lymph node involvement, which is close tour results.

The study by Algebally et al. [14] showed 57\% sensitivity, $67.3 \%$ specificity, and $81.2 \%$ accuracy of MRI in assessment of lymph node involvement.

Lee et al. [19] stated that with MRI scans they were able to gain $80 \%$ sensitivity and $92 \%$ accuracy for a patient-bypatient evaluation of nodal disease.

The status of the CRM is an important independent prognostic factor in the evaluation of rectal cancer. A free margin reduces the risk of local recurrence and improves survival. A distance of $1 \mathrm{~mm}$ between the mesorectal fascia and the primary tumor, lymph nodes with suspicious criteria and extramural vascular invasion considered affected CRM. Tumor extension near but $>1 \mathrm{~mm}$ distant from the circumferential resection margin is sometimes referred to as a threatened circumferential resection margin. [21]

Our results showed MRI sensitivity of CRM assessment reaching 96\%, specificity 94\% and accuracy 96\%. Algebally et al. [14] also reported $84 \%$ sensitivity, $97 \%$ specificity and $94 \%$ accuracy.

Our results are close to the results of Al-Sukhni et al. [21] that were $95 \%$ specificity and $93 \%$ sensitivity.

C. C. Moreno [17] stated that MRI is less accurate, being $74 \%$ sensitive and $88 \%$ specific for involvement of the mesorectal fascia by tumor. Also Rao S-X et al [13] states that MRI is $80 \%$ sensitive, $90.4 \%$ specific, and $88 \%$ accurate for prediction of mesorectal fascia involvement.

\section{Conclusion}

Our study demonstrated that High-resolution MRI is a highly accurate modality for prediction of transmural tumor invasion, mesorectal fascia invasion, adjacent organ invasion, pelvic lymph node involvement and CRM involvement. Accurate knowledge of those factors is essential for preoperative decision-making in rectal cancer treatment.
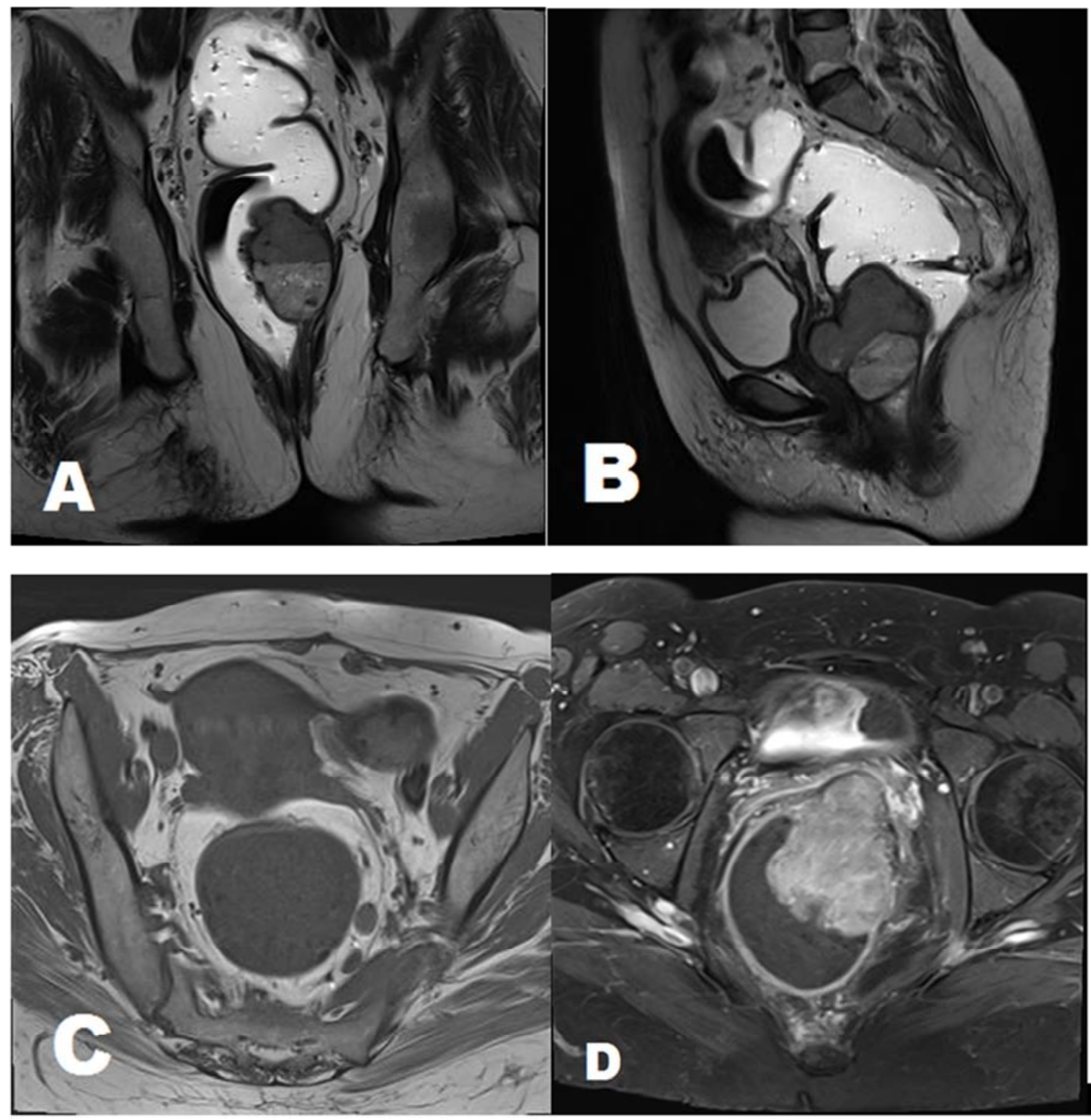


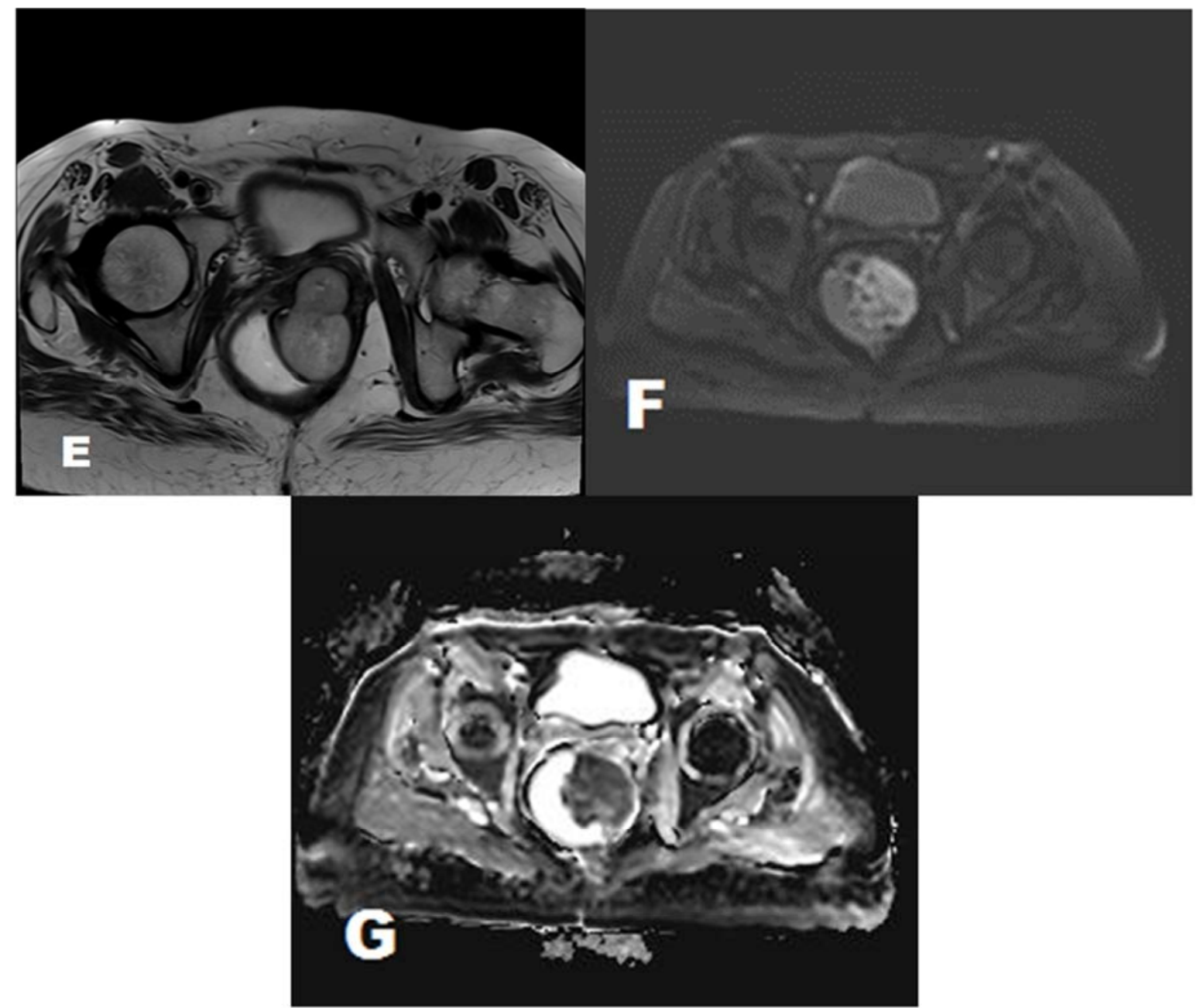

Figure 2. (A)Coronal T2 WI: Revealed a large endo-luminal fungating mass at the lower third of the rectum. Distance of the lower edge of the tumor to superior edge of internal anal sphincter about $1.5 \mathrm{~cm}$ as in image. (B)-Sagittal T2 WI: Distance of the lower edge of the tumor to the anal verge is about $5 \mathrm{~cm}$. (C) Axial T1 WIs: Illustrates rectal tumor, which appears isointense to muscles in T1 WIs, Also multiple mesorectal lymph nodes (LNs) of malignant criteria are seen $3 \mathrm{~mm}$ away from the mesorectal fascia (MRF). (D) Axial fat suppression post contrast T1 WIs: The rectal mass shows intense and heterogeneous contrast enhancement as well as invasion of the vagina. (E) Axial T2 WI: Demonstrates the extension of the rectal mass to the vaginal yet no signs of urinary bladder invasion. (F) Axial DWI: The rectal mass presenting hyperintense signal. (G) Axial ADC map: The rectal mass exhibited diffusion restriction pattern.

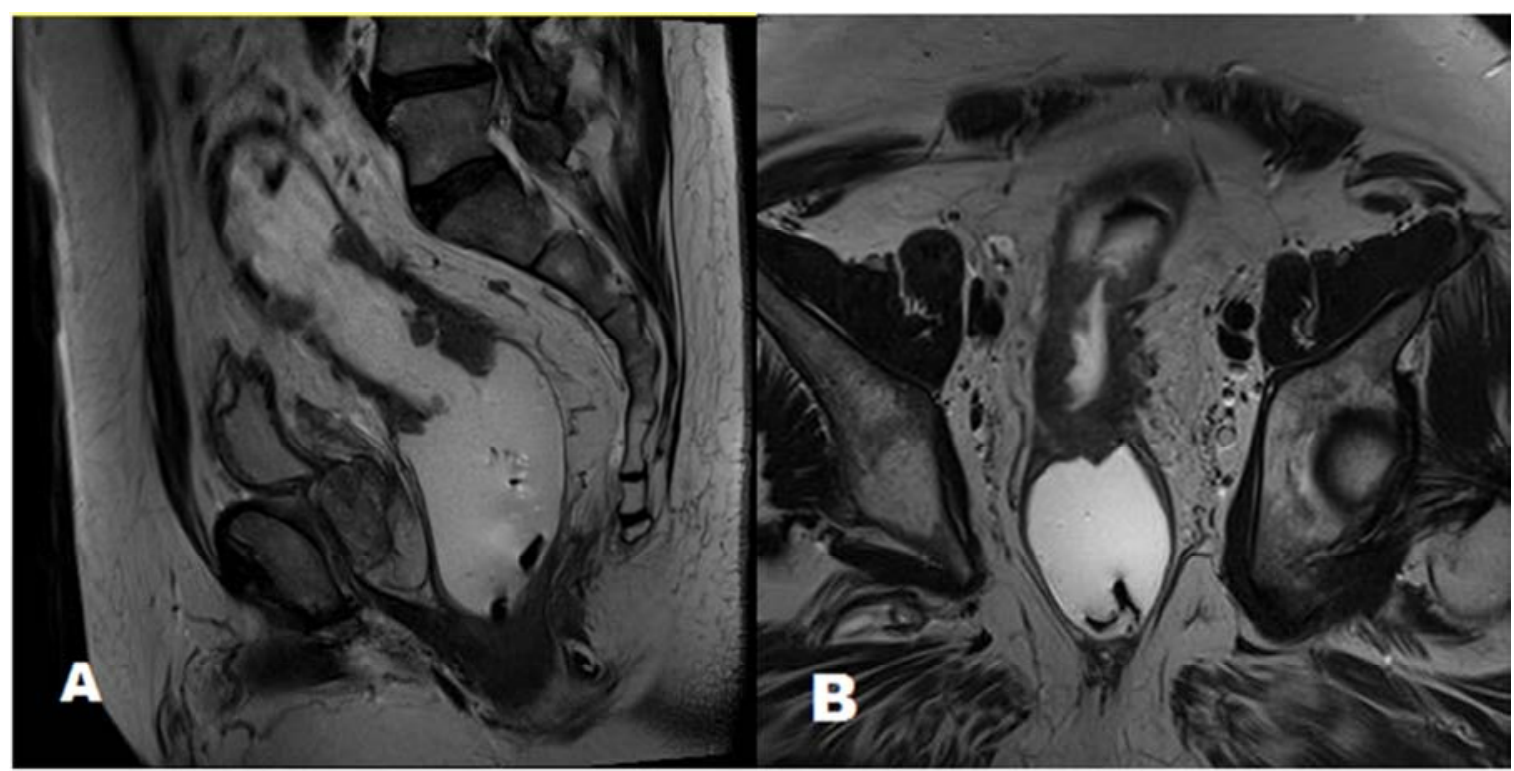



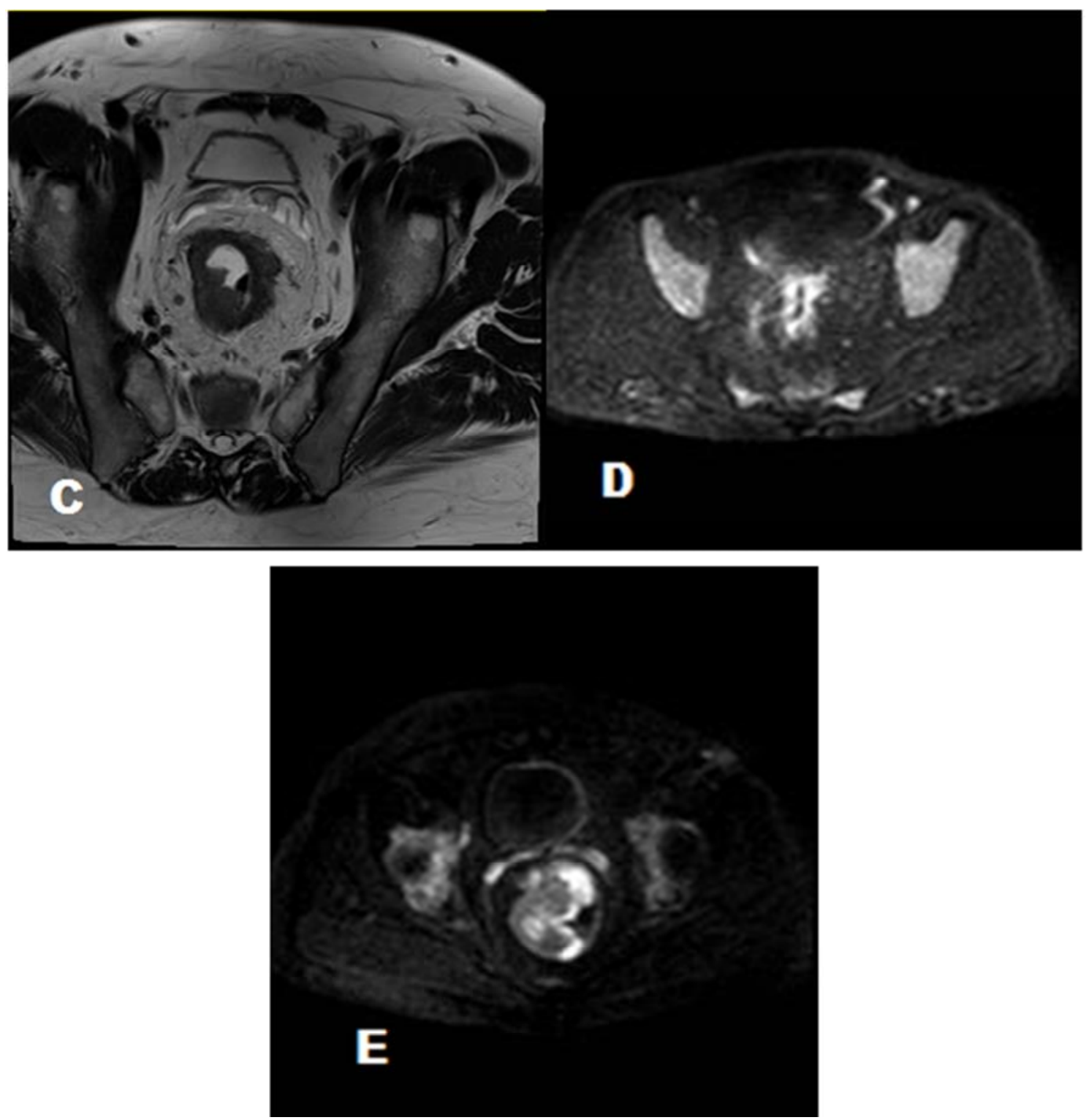

Figure 3. (A) Sagittal T2 WI: Demonstrates the site of the rectal tumor at the middle and the upper third of the rectum. (B) Coronal T2 WI: Distance of the lower edge of the tumor to superior aspect of internal anal sphincter about $4 \mathrm{~cm}$ as in image. (C)- Axial T2 WIs: Revealed circumferential irregular mural thickening with luminal narrowing. Invasion of muscularis propria is noted and maximum tumoral extrusion reaches about 6 mm. Multiple mesorectal lymph nodes are seen representing no malignant criteria and not exceeding $4 \mathrm{~mm}$ in size. Minimum distance of tumor from CRM 1.4 mm noted. (D) Axial DWI : The rectal mass presenting hyperintense signal. (E) Axial ADC map: The rectal mass exhibited diffusion restriction pattern.

\section{References}

[1] Siegel R, Naishadham D, Jemal A. Cancer statistics, CA Cancer J Clin 2013; 63: 11-30.

[2] Winawer SJ, Fletcher RH, Miller L, et al. Colorectal cancer screening: clinical guidelines and rationale. 1997: 12: 594-642.

[3] D. M. Ganeshan, et al. MRI Evaluation of Rectal Carcinoma, Cross-Sectional Imaging of the Abdomen and Pelvis: A Practical Algorithmic Approach, New York: Springer, 2015: 417-433.

[4] Klessen, C., Rogalla, P., \& Taupitz, M. Local staging of rectal cancer: the current role of MRI. European radiology 2007; 2: 379-389.
[5] Ümit Tapan, Mustafa Özbayrak, Servet Tatlı. MRI in local staging of rectal cancer: an update. Diagnostic Interventional Radiology 2014; 20: 390-398.

[6] Suk Hee Heo, Jin Woong Kim, Sang Soo Shin, et al. Multimodal imaging evaluation in staging of rectal cancer. world journal of gastroenterology 2014; 15: 4244-4255.

[7] Harmeet K, Haesun C, Y Nancy Y, et al. MR Imaging for Preoperative Evaluation of Primary Rectal Cancer: Practical Considerations radioGraphics 2012; 3: 389-409.

[8] J. Evans, U. Patel, and G. Brown. Rectal Cancer: Primary Staging and Assessment After Chemoradiotherapy Seminars on Radiation Oncology 2013; 21: 169-177.

[9] Brown G, Richards CJ, Newcombe RG, et al. Rectal carcinoma: thin-section MR imaging for staging in 28 patients. Radiology. 1999; 211: 215-222. [PubMed] 
[10] Shihab OC, Heald RJ, Rullier E, et al. Defining the surgical planes on MRI improves surgery for cancer of the low rectum. Lancet Oneal. 2009; 10: 1207-1211. [PubMed]

[11] H. Kwok, I. P. Bissett, G. L. Hill. Preoperative staging of rectal cancer. International Journal of Colorectal Disease. 2000; 15: 9-20

[12] Elizabeth Furey, Kartik S, Jhaveri. Magnetic resonance imaging in rectal cancer. Clinics Northern America 2014; 22: 165-190.

[13] Rao S-X, Zeng M-S, et al. Assessment of $\mathrm{T}$ staging and mesorectal fascia status using high-resolution MRI in rectal cancer with rectal distention. World J Gastroenterol. 2007; 13: 4141-4146.

[14] Algebally AM et al. The value of high-resolution MRI technique in patients with rectal carcinoma: pre-operative assessment of mesorectal fascia involvement, circumferential resection margin and local staging. Pol J Radiol. 2015; 80: $115-21$

[15] Matsuoka H, Nakamura A, Masaki T, et al. Optimal diagnostic criteria for lateral pelvic lymph node metastasis in rectal carcinoma. Anticancer Res. 2007; 27: 3529-3533
[16] Wong, et al. Effect of endorectal coils on staging of rectal cancers by magnetic resonance imaging. Hong Kong Med J 2010; 16: 421-439.

[17] C. C. Moreno, et al. Magnetic resonance imaging of rectal cancer: staging and restaging evaluation. World J Gastroenterol 2014; 15: 4244-4255.

[18] Zhang XM, Zhang HL, et al. 3-T MRI of rectal carcinoma: preoperative diagnosis, staging and planning of sphinctersparing surgery. AJR Am J Roentgenol 2008; 5: 1271-8.

[19] HY. Lee et al. Prognostic Significance of Metastatic Lymph Node Ratio in Node-Positive Colon Carcinoma. Annals of Surgical Oncology 14; 5: 1712-1717.

[20] Brown G, Richards CJ, Bourne MW, et al: Morphologic predictors of lymph node status in rectal cancer with use of high-spatial-resolution MR imaging with histopathologic comparison. Radiology 2003; 2: 371-377.

[21] Eisar Al-Sukhni, Laurent Milot, Mark Fruitman, et al. Diagnostic Accuracy of MRI for Assessment of T Category, Lymph Node Metastases, and Circumferential Resection Margin Involvement in Patients with Rectal Cancer: A Systematic Review and Meta-analysis. Ann Surg Oncol. 2012; 19: 2212-2223. 